\title{
Final Declaration of the Asian Metaforum on the Role of Vitamin D and the Management of Osteoporosis
}

\author{
Siew-Pheng Chan, Jung-Fu Chen, Leung-Wing Chu, Dai-Phi Van, David Hosking, \\ Tai-Pang Ip, Leonard Koh, Annie Kung*, Ning-Sheng Lai, Edith Lau, Joon-Kiong Lee, \\ Rattana Leewattana, Yong-ki Min, Nguyen-Dac Nghia, Ongphiphadhanakul Boonsong, \\ Hyoung-moo Park, Johann Ringe, Bambang Setyohadi, Chan-Soo Shin, Suppasin \\ Soontrapa, Nimit Taechakraichana, Fachry Tanjung, Dohar Tobing, \\ Keh-Sung Tsai, Jean Woo and Rong-Sen Yang \\ c/o Department of Medicine, University of Hong Kong, Queen Mary Hospital, Pokfulam, Hong Kong Special \\ Administrative Region, People's Republic of China
}

Submitted 17 August 2007: Accepted 7 May 2008: First published online 23 July 2008

\begin{abstract}
Background: Vitamin D is essential for Ca absorption, prevention of falls and fracture, and maintenance of muscle strength and balance. Lack of awareness of the importance of vitamin D in bone health is common in Asia.

Objective: To define key statements, objectives and actions for improving osteoporosis management and vitamin D inadequacy in Asia.

Results and conclusion: This declaration was jointly produced by specialists at the Asia Metaforum on the Role of Vitamin D and the Management of Osteoporosis, held in September 2006 in Hong Kong, to define actions to prevent vitamin D insufficiency in Asia. Although developed specifically for Asia, some or all of these statements may be applicable to other regions of the world.
\end{abstract}

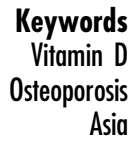

At the Asian Metaforum on the Role of Vitamin D and the Management of Osteoporosis held on 23-24 September 2006 in Hong Kong, we, the participants, discussed and presented data on the management of osteoporosis and the epidemiology and clinical consequences of vitamin D inadequacy in Asia. The main outcome and goal of the Metaforum were to produce a Declaration on the role of vitamin $\mathrm{D}$ in bone health and disease prevention, particularly with respect to osteoporosis. This Declaration defines key statements, objectives and actions for improving osteoporosis management and vitamin D inadequacy in Asia, as well as criteria for measuring success.

1. Vitamin D is essential for Ca absorption and bone health in men and women. Vitamin D inadequacy determined by serum 25-hydroxyvitamin D [25(OH)D] levels - may lead to suboptimal Ca absorption, secondary hyperparathyroidism with high bone turnover and an increased risk of fracture, especially among those with osteoporosis and in older people ( $\geq 65$ years). In Asian clinical practice, it is very common to find patients with vitamin D inadequacy, such that widespread treatment is necessary. However, measurement of serum $25(\mathrm{OH}) \mathrm{D}$ levels is not routinely performed.
Objective:

- To ensure that all osteoporosis patients receive, at the very least, the recommended daily allowance (RDA) of vitamin D on a regular basis.

Actions:

- Identify the facts that will help women and men, as well as their physicians, to understand the importance of vitamin D in the management of osteoporosis.

- Explain the links between vitamin D and Ca absorption.

Measure of success:

- All osteoporosis patients should receive at least the RDA of vitamin D on a regular basis. Success will be monitored by the prescription volume of vitamin D in osteoporosis patients.

2. Vitamin $D$ is particularly important for maintaining muscle function and balance. Vitamin D inadequacy may contribute to the risk of falls and fracture.

Objective:

- To reduce the risk, and improve the management, of falls in osteoporosis patients. This would include increasing awareness about the importance of vitamin $\mathrm{D}$ for bone health and the prevention of fracture. 
Actions:

- Establish falls-prevention programmes within fracture management protocols.

- Incorporate advice on avoiding vitamin D inadequacy in these fracture management protocols.

- Increase the number of fall-prevention programmes in each Asian country.

- Ensure that the majority of osteoporosis guidelines in Asia incorporate guidance on fall management.

- Ensure that the evaluation of falls is incorporated into the assessment of every fracture patient.

- Increase the percentage of osteoporosis patients who are enrolled in fall-prevention programmes.

Measure of success:

- Success will be monitored by the incidence of falls in in-patient and out-patient records.

- The percentage of guidelines in Asia that includes fall prevention.

- The percentage of osteoporosis patients who have received education and rehabilitation on fall prevention.

3. The main source of vitamin D is sunlight and very little comes from food. The low $\mathrm{Ca}$ intake of many Asian populations makes the need to ensure vitamin D adequacy an even greater priority.

Objective:

- To increase vitamin D levels of the general population.

Actions:

- Educate doctors and patients about the importance of sunlight for vitamin D nutrition.

- Address cosmetic and cultural barriers to increasing sunlight exposure.

- Use supplements to achieve the RDA of vitamin D in those with insufficient vitamin D from sunlight or food.

- Ensure that all patients with osteoporosis receive vitamin D supplementation unless they are clearly vitamin D replete.

Measure of success:

- Evaluate the percentage that incorporates information on vitamin D in a random selection of patient education materials.

- Monitor the prescription volume of vitamin D in osteoporosis patients and patient's compliance to treatment.

4. Vitamin D inadequacy in Asia is widespread among both the young and the old, regardless of location, season or climate. There may be variations between ethnic groups, but the problem of inadequacy is highly prevalent, particularly in people with an urbanized lifestyle.

Objective:

- To increase vitamin D supplementation among osteoporosis patients and at-risk individuals within the community.
Actions:

- Raise physician awareness of the high prevalence of vitamin D inadequacy and the need to ensure that it is corrected. The key points are:

O The prevalence of vitamin D inadequacy is very high, regardless of geographical location and climate.

- Vitamin D provides multiple benefits for muscle function, prevention of falls and bone health.

- Vitamin D is safe and simple to incorporate within an effective treatment plan.

- Encourage patients to consider the kind of lifestyle modifications that could help in primary prevention.

o For example, encourage women of working age to ensure moderate levels of sun exposure required for vitamin D sufficiency.

Measure of success:

- In patients with osteoporosis, at least double the percentage who take vitamin D by the end of 2010 . This should be measured by the prescription volume of vitamin D and patients' compliance to treatment.

5. Vitamin D is an essential part of osteoporosis management. It enhances the effectiveness of prescribed medicine and $\mathrm{Ca}$ supplements for the prevention of fractures.

Objective:

- To increase vitamin D supplementation at osteoporosis management centres.

Action:

- Identify the key facts that will help women and men, and their physicians, to understand the importance of vitamin $\mathrm{D}$ in the management of osteoporosis. The key points are:

$\circ$ Vitamin D is essential to help prevent falls and fractures.

- Sufficient amounts of Ca cannot be absorbed without vitamin D.

- Most people do not get enough vitamin D from food and sunlight, the natural sources of vitamin D.

- Almost all osteoporosis patients should receive vitamin $\mathrm{D}$ as part of their treatment, alongside an effective prescribed medicine.

- Taking vitamin D is safe.

- Measure the level of, and compliance with, vitamin D supplementation in major osteoporosis centres.

Measure of success:

- Monitor the prescription volume of vitamin D supplementation and patient's compliance to treatment.

- Recommendation on the use of vitamin D plus an osteoporosis medicine with proven efficacy for preventing fracture in all national/regional clinical management guidelines for health-care professionals by 2010 in Asia. Success will be monitored by surveying these guidelines in 2010 for the presence of these recommendations. 
6. All patient and professional organizations supporting patients with osteoporosis should provide current information on the critical role of vitamin $\mathrm{D}$ in reducing the risk of falls and fracture.

Objective:

- To ensure that all literature and websites of Asian osteoporosis patient groups disseminate accurate information about the importance of vitamin $\mathrm{D}$ and adequate $\mathrm{Ca}$ absorption.

Actions:

- Develop patient educational materials to explain the critical role of vitamin D in muscle function, bone health and osteoporosis management.

- Encourage sustained compliance with established national guidelines among medical professionals.

Measure of success:

- All literature and websites associated with Asian osteoporosis patient groups should include accurate guidance on the importance of vitamin $\mathrm{D}$ and adequate $\mathrm{Ca}$ absorption by the end of 2010. Success will be measured by evaluating the percentage that includes such information in a random selection of these literature and websites.

7. Adequate vitamin $\mathrm{D}$ intake is very important in the primary prevention of osteoporosis. It should start early in life and continue throughout life. A balanced diet providing adequate energy and protein, and daily weight-bearing exercise, are also very important.

Objective:

- To increase awareness of the biological functions of vitamin D among children of school age and among adults.

Action:

- Include education about osteoporosis prevention in schools and in general health education programmes for adults.

Measure of success:

- This will be measured by the percentage that includes information on vitamin $\mathrm{D}$ in a random selection of educational materials for children and adults.

\section{Conclusion}

Vitamin D is essential for Ca absorption and for maintaining bone health and muscle function. However, there is currently a high prevalence of vitamin D insufficiency in Asia - irrespective of location and climate - which threatens to reach epidemic proportions. The consequences of vitamin D inadequacy include suboptimal Ca absorption, secondary hyperparathyroidism with high bone turnover and increased fracture risk, particularly among those with osteoporosis and the elderly ( $\geq 65$ years). The implications of vitamin $\mathrm{D}$ inadequacy on osteoporosis outcomes are clear and vitamin D should be an essential component of osteoporosis management. Ultimately, education on vitamin D targeted at every level of the population - osteoporosis patients, treating physicians and associated health-care professionals, children, the elderly and those at risk of osteoporosis - via a variety of media, health education programmes and disease management protocols is recommended. These educational measures will play a major role to improve vitamin D status, and osteoporosis prevention and management. The effectiveness of simple lifestyle modifications, such as improving diet, increasing weight-bearing exercise and greater sunlight exposure, should be communicated and emphasized, and all physicians should ensure that their patients with, or at risk of, osteoporosis should regularly receive at least the RDA of vitamin D. Finally, although this Declaration has been developed specifically for Asia, some or all of its statements may be relevant for describing the state of vitamin D inadequacy and osteoporosis management in other world regions. As such, this Declaration may be useful for other regions aiming to improve vitamin D status or osteoporosis prevalence among their populations. Ultimately, the overall effect of such region-wide consensus would be a more global push for increasing awareness of vitamin D inadequacy and its serious health consequences.

\section{Acknowledgements}

The authors have no conflicts of interest. The authors spent two days attending a meeting to develop this declaration. This project is supported by Merck Sharp \& Dohme (Asia) Ltd. All authors were present at the Asian Metaforum on the Role of Vitamin D and the Management of Osteoporosis held on 23-24 September 2006 in Hong Kong; participated, discussed and presented data on the management of osteoporosis and the epidemiology and clinical consequences of vitamin D inadequacy in Asia; and contributed equally in drafting this declaration. 